\title{
Spiral shaped microorganisms in the human duodenal mucosa
}

\author{
I.M. Nakshabendi' ${ }^{1}$ S.E. Peebles ${ }^{2}$, F.D. Lee ${ }^{2}$ and R.I. Russell ${ }^{1}$ \\ Department of ${ }^{1}$ Gastroenterology and ${ }^{2}$ Pathology, Royal Infirmary, Glasgow G31 2ER, UK
}

Summary: A new spiral shaped microorganism, Gastrospirillum hominis, distinct from Helicobacter pylori, has recently been described in the gastric mucosa. We report a patient with duodenal erosions who was found to have these organisms in his duodenal mucosa. This bacterium is not necessarily specific to the stomach, and its association with peptic damage needs to be studied further.

\section{Introduction}

Following the discovery of Helicobacter pylori ${ }^{1,2}$ and its pathological significance in the upper gastrointestinal tract, another microorganism has been described and later named Gastrospirillum hominis. ${ }^{3,4}$ This Gram-negative organism is also spiral shaped, and until now has been found only in the stomach. ${ }^{5,6}$ We describe a case in which $G$. hominis was found in the duodenal mucosa, and may have been associated with symptoms.

\section{Case report}

A 44 year old salesman presented with a 6-month history of heartburn and epigastric discomfort. He had had similar episodes 8 years ago and a barium meal then was reported as being normal. He smoked 10 cigarettes and drank 12 units of alcohol per week. Physical examination revealed epigastric tenderness only. Routine biochemical and haematological tests were normal. At endoscopy the oesophagus and stomach were normal: in the first part of the duodenum there were multiple erosions $1-3 \mathrm{~mm}$ in diameter, surrounded by mucosal erythema. These erosions were on the tip of polypoidal protrusions from the mucosa and were biopsied to exclude any unusual pathology. The duodenal biopsies were processed in paraffin wax and sections were stained with haematoxylin and eosin. Features of active duodenitis with gastric metaplasia and Brunner's gland hyperplasia were seen. A cluster of microorganisms with spiral

Correspondence: I.M. Nakshabendi, M.R.C.P. Accepted: 9 January 1991 configuration was also identified within free-lyingo mucoid material adjacent to areas of gastric metaplasia. Each microorganism was approximatelyes $5 \mu \mathrm{m}$ in length, blunt-ended and had 5-6 longitudinal twists (Figure 1). The appearances of these $\frac{\mathbb{D}}{3}$ microorganisms were in keeping with those $\overline{0}$

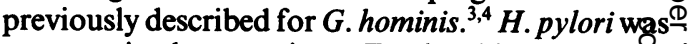
not seen in these sections. Further biopsies taken $\overrightarrow{0}$ from the gastric antrum and duodenum were foug $d$ to be positive for urease (CLO test). The patie was empirically given tripotassium dicitratobis-O muthate (De-Noltab) $480 \mathrm{mg} /$ day for 4 weeks together with metronidazole $600 \mathrm{mg} /$ day and amoxycillin $750 \mathrm{mg} /$ day for 2 weeks. He was $\frac{\%}{\circ}$ reviewed at the outpatient clinic 4 weeks later. All his symptoms had disappeared but he refused too를 have a repeat endoscopy to obtain more biopsies to check his response to the above therapy.

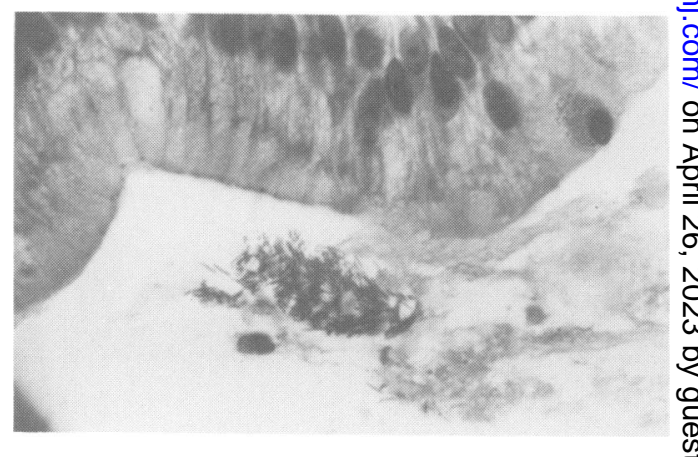

Figure 1 Duodenal biopsy (magnification $\times 1254)$ ? showing a cluster of the spiral-shaped microorganism, Gastrospirillum hominis. 


\section{Discussion}

The observations in this patient suggest that $G$. hominis is capable of colonizing the duodenal mucosa. Ideally we should have looked for these organisms in the gastric mucosa as well. The positive CLO test in his stomach made us believe that the patient had $H$. pylori, and the duodenum was biopsied only to exclude unusual pathology. It is interesting to find that the duodenal biopsies were positive for both urease activity and $G$. hominis. $H$. pylori was not seen in the duodenum. This might suggest that $G$. hominis is capable of producing urease, confirming previous reports. ${ }^{5,6}$ The pathological significance of $G$. hominis is not fully clear, but in our case it appears to have been associated with duodenal erosions. Previous studies reported the presence of active chronic gastritis, gastric ulceration and oesophagitis in association with these organisms, but the duodenum was found to be normal at endoscopy ${ }^{5,6}$ and histology. ${ }^{5}$ Our patient is, therefore, the first to be described as having duodenal endoscopic and histological abnormalities with the presence of $G$. hominis.

The association of the above pathological changes and $G$. hominis does not necessarily imply a cause and effect phenomenon. It is worth noting that $G$. hominis is a rare organism. It was found in 6 out of 1650 cases in one report ${ }^{5}$ and in 2 out of 700 subjects in another series. ${ }^{6}$ The precise role of such an organism in a common but multifactorial disease, such as peptic ulceration, remains to be further studied.

\section{References}

1. Warren, J.R. Unidentified curved bacilli on gastric epithelium in active chronic gastritis. Lancet 1983, i: 1273.

2. Marshall, B.J. Unidentified curved bacilli on gastric epithelium in active chronic gastritis. Lancet 1983, i: 1273-1275.

3. Dent, J.C., McNulty, C.A.M., Uff, J.C., Wilkinson, S.P. \& Gear, M.W.L. Spiral organism in the gastric antrum. Lancet 1987, ii: 96.

4. Editorial. Gastrospirillum hominis. Lancet 1989, ii: 252-253.

5. McNulty, C.A.M., Dent, J.C., Curry, A. et al. New spiral bacterium in gastric mucosa. J Clin Pathol 1989, 42: 585-591.

6. Morris, A., Rafiq Ali, M., Thomsen, L. \& Hollis, B. Tightly spiral shaped bacteria in the human stomach: another cause of active chronic gastritis? Gut 1990, 31: 139-143. 